\title{
Topical carbonic anhydrase inhibition increases ocular pulse amplitude in high tension primary open angle glaucoma
}

\author{
Karl-Georg Schmidt, Andrea von Rückmann, Lutz E Pillunat
}

\begin{abstract}
Background-Ocular pulse amplitude (OPA) is reduced in normal tension primary open angle glaucoma (NTP) patients when compared with healthy age matched controls (CTL) while increased OPA appears to protect ocular hypertensive patients from visual field loss. If NTP is accompanied by vasospasm, as in roughly half of the primary open angle glaucoma (POAG) population (independent of intraocular pressure, IOP), calcium channel blockers increase OPA and thus stabilise visual fields in these patients. Current glaucoma drugs reduce IOP but do not activate (compromised) ocular perfusion.
\end{abstract}

Methods-The influence of dorzolamide, a topical carbonic anhydrase inhibitor in standard dosage (three times daily, one eye) on OPA, IOP, blood pressure, and heart rate was investigated in a randomised, prospective, masked clinical trial assessing the acute effects of dorzolamide $v$ placebo before and 2 days after application in 33 cataract patients with (n $=14)$ and without $(n=19)$ high tension POAG (HTP) who provided informed consent.

Results-Following application of dorzolamide (D) IOP (mm Hg, mean (SEM)) in $\mathrm{HTP}_{\mathrm{D}}(20.2(0.5) / 16.3(0.5))$ and in $\mathrm{CTL}_{\mathrm{D}}$ (16.0 (0.5)/12.3 (0.5)) was highly significantly $(p<0.001)$ reduced and was significantly $(p<0.03)$ reduced in vehicle $(V)$ treated eyes $\left(\right.$ HTP $\left._{\mathrm{v}}: 20.3(0.4) / 19.0(0.4)\right)$ and $\mathrm{CTL}_{\mathrm{v}}$ : $\left.15.8(0.4) / 14.9(0.3)\right)$ when compared with respective baseline measurements. OPA (mm Hg) in HTP $_{\mathrm{D}}(2.1$ $(0.1) / 2.5(0.1))$ and $\mathrm{CTL}_{\mathrm{D}}(2.2(0.1) / 2.6$ $(0.2))$ eyes was significantly $(p<0.05)$ increased and unaffected in vehicle treated eyes when compared with respective baseline measurements. Systemic perfusion variables were also unchanged.

Conclusion-Dorzolamide increased OPA in HTP and CTL. Drugs stimulating OPA may improve prognosis of POAGs.

(Br f Ophthalmol 1998;82:758-762)

A reduction of ocular pulse amplitude (OPA) in primary open angle glaucoma (POAG) patients with normal tension $(\mathrm{NTP})^{1-4}$ independent of vasospasm ${ }^{4}$ seems to correlate with progression of visual field loss, ${ }^{5}$ whereas increased OPA appears to protect ocular hypertensive patients from visual field loss and glaucomatous disc cupping, which we interpret as a protective mechanism or adaptive change to prevent hypoperfusion induced damage to optic nerve fibre layers. ${ }^{12}$

OPA is the measure of a bolus of blood entering the eye, the maximum and minimum measurements reflect, in keeping with the cardiovascular definitions of systolic and diastolic blood pressures, systolic and diastolic intraocular pressures (IOPs), respectively, ${ }^{6}$ which illustrate the pulsatile component of intraocular blood flow.

According to $\mathrm{Alm}^{8}$ total ocular perfusion as determined in monkeys is approximately 800 $\mathrm{mg} / \mathrm{min}$, the choroid contributes $677 \mathrm{mg} / \mathrm{min}$ $(85 \%)$, the iris $8 \mathrm{mg} / \mathrm{min}(1 \%)$, the ciliary body $81 \mathrm{mg} / \mathrm{min}(10 \%)$, and the retina $34 \mathrm{mg} / \mathrm{min}$ $(4 \%)$. The pulsatile component of the choroidal perfusion as investigated by colour coded Doppler ultrasonography is approximately $70 \% .^{9}$

The ocular blood flow $(\mathrm{OBF})$ system $(\mathrm{OBF}$ Labs Ltd, Wilts), a highly sensitive pneumotonometer linked to a data acquisition unit measures IOP ( $\mathrm{mm} \mathrm{Hg}$ ) non-invasively with a collection rate of $200 \mathrm{~Hz}$, allowing accurate, non-invasive, and reproducible ${ }^{1011}$ measurements of OPA with minimal disturbance to the eye.

Ocular blood flow was not calculated from OPA measurements because extraocular factors are involved (for example, pulse rate and posture) and, in our opinion, the underlying assumptions (pressure/volume relation in the living human eye) as well as the lack of relevant intraocular factors (width of pulse amplitude, scleral rigidity, axial length) and the software provided with the $\mathrm{OBF}$ system are not adequately documented to calculate blood flow from the pulse data.

Even if the goal of current glaucoma therapy (high tension POAG, HTP: IOP $<20 \mathrm{~mm} \mathrm{Hg}$; NTP: IOP $<15 \mathrm{~mm} \mathrm{Hg}$ ) is reached, progression of visual field loss and optic nerve head cupping continue in many subjects, mandating that therapeutic concepts independent of or in addition to reducing IOP be considered. One of these is increasing ocular blood flow.

Systemic carbonic anhydrase inhibitors (CAIs) such as acetazolamide are increasingly being used as vasodilators, equally effective as carbon dioxide, to detect cerebrovascular vasomotor reserve capacity and increase cerebral blood flow and oxyhaemoglobin in patients with chronic cerebrovascular disease. ${ }^{12-14}$ Thus, CAI has been shown to improve visual function probably by increasing ocular pulse 
volume and pulsatile ocular blood flow $^{15}$ induced by a reduction in $\mathrm{pH}$ of blood and tissues. ${ }^{16}$

The long term use of this systemic drug is limited by side effects such as electrolyte imbalance, fatigue, depression, gastrointestinal complaints, occurrence of renal stones as well as blood dyscrasias.

The present study was designed to evaluate for the effect of dorzolamide hydrochloride (Trusopt, Merk \& Co Inc, West Point, PA, USA) a topical CAI on OPA in HTP patients and non-glaucomatous controls (CTL).

\section{Materials and methods}

PATIENTS

Sixteen CTL subjects (nine male, seven female), 46-68 (mean 58.0) years of age with no known ocular disease other than cataract, who showed no visual field defects and/or disc alterations and had IOPs $<21 \mathrm{~mm} \mathrm{Hg}$ and 12 patients with bilateral HTP and cataracts (five male, seven female), 42-71 (mean 56.3) years of age, each undergoing cataract surgery received dorzolamide in the one eye undergoing cataract surgery in standard dosage (three times daily), hydroxyethylcellulose (three times daily) as a placebo in the contralateral control eye, and complied with the protocol of this study. Additionally, both eyes of HTP and CTL patients received gentamicin eye drops six times per day as preoperative antibiotic prophylaxis.

The HTP and CTL patients had no history of systemic hypertension, diabetes mellitus, elevated cholesterol, neurological deficits, or typical migraine headache, and had no known eye disease other than the category selected for or ocular surgery including laser of any kind and remained on their systemic therapy. The HTP patients chosen had a history of pretreatment IOPs $>21 \mathrm{~mm} \mathrm{Hg}$, had typical glaucomatous disc cupping (disc size $1.8-2.3 \mathrm{~mm}^{2}, \mathrm{C} / \mathrm{D}$ ratio $>0.6$ ) with corresponding visual field loss, remained on their long standing, unaltered (>3 months) ocular hypotensive therapy, and additionally received dorzolamide.

Only HTP patients on either adrenaline $(1 \%)$ or timolol $(0.5 \%)$, eye drops which have been shown to reduce IOP, but not to affect

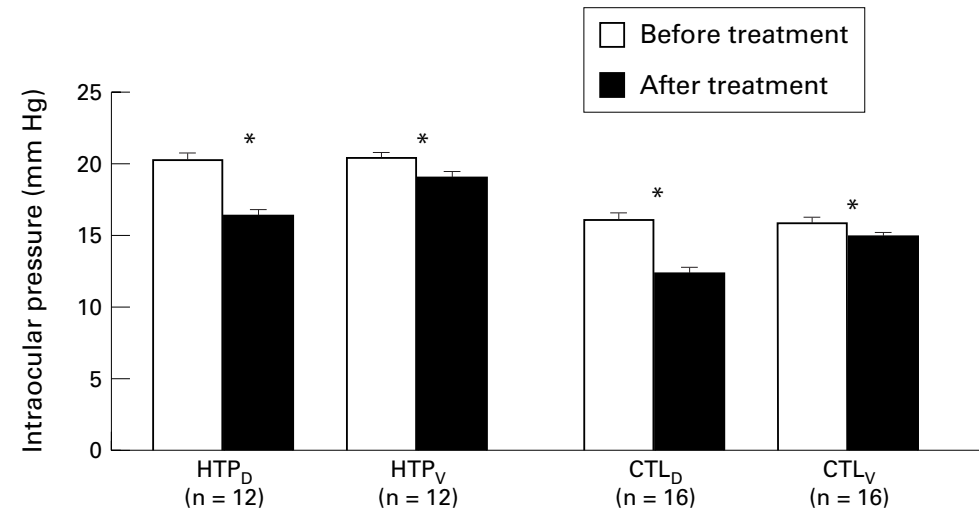

Figure 1 Intraocular pressure (IOP) in dorzolamide (D) and vehicle (V) treated eyes of high tension primary open angle glaucoma $(H T P)$ patients $(n=12)$ and non-glaucomatous controls (CTL, $n=16$ ) before and after drug or vehicle application. Bars represent mean (SEM). *Indicates $p<0.05$ compared with measurements before drug or vehicle application using the Student's two tailed, paired t test.
OPA in our human studies ${ }^{12}{ }^{17}$ were allowed in this trial; patients continued these medications in their usual dosage during the course of the trial.

STUDY PROTOCOL

In this study dorzolamide $(2 \%)$ and hydroxymethylcellulose as a vehicle were each applied three times daily to either eye of each patient for 2 days.

In addition to pneumotonometric values, IOP was measured by Goldmann applanation tonometry. Brachial artery pressures were measured non-invasively (Riva-Rocci) using a blood pressure cuff and a stethoscope. Heart rate (HR) was measured manually using a stopwatch. Applanation IOP, systolic (BPsyst) and diastolic (BPdiast) blood pressures (BP), and HR were taken before OPA measurements.

All OPA and IOP readings for this study were taken by the same examiner, who performed these measurements masked to which eye was drug treated.

Measurements of patients were taken before and during dorzolamide treatment with the patient in a seated position at the same time of day with respect to pre-dorzolamide measurements and 2 hours after drug and vehicle application.

The protocol was accepted by the institutional review board of the University of Giessen and informed consent was obtained from each patient following complete explanation of the procedure.

\section{STATISTICS}

All data were normally distributed and represent arithmetic mean (plus or minus SEM); Student's paired two tailed $t$ test was used for statistical analysis. Measurements were performed on both eyes of each subject. One eye received dorzolamide, the other eye was vehicle treated. Pretreatment values did not differ significantly in treated versus untreated HTP and CTL groups, respectively. Data before treatment were compared with data from respective groups after 2 days of dorzolamide treatment before surgery. The level of significance was $5 \%$ - that is, $\mathrm{p}<0.05$ was considered significant.

\section{Results}

Following administration of dorzolamide (D) IOP $(\mathrm{mm} \mathrm{Hg})$ was significantly $(\mathrm{p}<0.001)$ reduced in drug treated $\mathrm{HTP}\left(\mathrm{HTP}_{\mathrm{D}}\right)$ patients, from $20.2(0.5)$ to $16.3(0.5)$, reflecting a $19.3 \%$ reduction of IOP. After the administration of the same topical drug using the protocol mentioned above IOP was significantly $(\mathrm{p}<0.001)$ reduced in drug treated CTL subjects $\left(\mathrm{CTL}_{\mathrm{D}}\right)$, from $16.0(0.5)$ to $12.3(0.5)$, reflecting a $23.1 \%$ reduction of IOP (Fig 1 ). In vehicle treated HTP $\left(\mathrm{HTP}_{\mathrm{v}}\right)$ IOP was significantly $(\mathrm{p}<0.03)$ reduced from $20.3(0.4)$ to $19.0(0.4)$, corresponding to a $6.4 \%$ reduction of IOP (Fig 1). In vehicle treated CTL $\left(\mathrm{CTL}_{\mathrm{v}}\right)$ IOP was significantly $(\mathrm{p}<0.03)$ reduced from $15.8(0.4)$ to $14.9(0.3)$, reflecting a $5.7 \%$ decrease in IOP (Fig 1). 


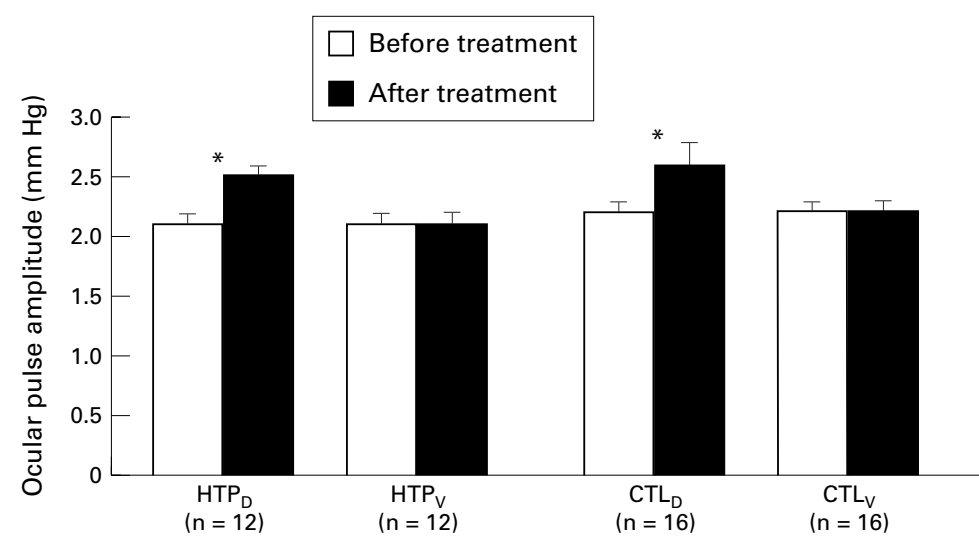

Figure 2 Ocularpulse amplitude (OPA) in dorzolamide (D) and vehicle $(V)$ treated eyes of high tension primary open angle glaucoma $(H T P)$ patients $(n=12)$ and non-glaucomatous controls (CTL, $n=16)$ before and after drug or vehicle application. Bars represent mean (SEM). ${ }^{\star}$ Indicates $p<0.05$ compared with measurements before drug or vehicle application using the Student's two tailed, paired t test.

Table 1 Systemic haemodynamic variables

\begin{tabular}{lcc}
\hline & Before treatment & During treatment \\
\hline BP (syst, mm Hg) & $132.3(9.4)$ & $129.3(9.4)$ \\
$\quad$ HTP & $134.6(7.1)$ & $131.0(6.9)$ \\
CTL & $81.3(6.3)$ & $78.8(6.5)$ \\
BP (diast, mm Hg) & $84.3(5.2)$ & $83.6(5.4)$ \\
$\quad$ HTP & $65.2(4.2)$ & $64.4(4.9)$ \\
CTL & $62.7(3.8)$ & $64.1(4.4)$ \\
Heart rate ( min $\left.^{-1}\right)$ & & \\
HTP & \\
CTL &
\end{tabular}

HTP $=$ high tension primary open angle glaucoma patients $(\mathrm{n}=12)$ and non-glaucomatous controls $(\mathrm{CTL}, \mathrm{n}=16)$.

Administration of dorzolamide caused a significant $(\mathrm{p}<0.05)$ increase of OPA $(\mathrm{mm} \mathrm{Hg})$ in $\mathrm{HTP}_{\mathrm{D}}$ from $2.1(0.1)$ to 2.5 (0.1), reflecting a $19.1 \%$ increment (Fig 2). After administration of the same topical drug using the protocol mentioned above in $\mathrm{CTL}_{\mathrm{D}}$, OPA increased from $2.2(0.1)$ to $2.6(0.2)$, reflecting an $18.2 \%$ rise (Fig 2). Following application of the vehicle using the protocol mentioned above OPA was unaffected in $\mathrm{HTP}_{\mathrm{V}}(2.1(0.1) / 2.1(0.1))$ and $\mathrm{CTL}_{\mathrm{v}}(2.2(0.1) / 2.2(0.1))$ eyes when compared with respective measurements before dorzolamide treatment (Fig 2).

Systemic perfusion variables were not significantly $(\mathrm{p}>0.05)$ altered in HTP and CTL subjects when compared with respective baseline measurements (Table 1).

\section{Discussion}

In treated eyes IOP reduction following application of topical dorzolamide $2 \%$ three times daily was 19.3 and $23.1 \%$ in HTP and CTL, respectively, which is in the range of other clinical trials $(18-30 \%)^{18-22}$; in the same eyes OPA increased 19.1 and $18.2 \%$ in HTP and CTL subjects, respectively, which correlates well with the IOP reduction suggesting an IOP/OPA interdependence in this range of IOPs.

Following the concept that IOP exerts a compressive force on the choroid, a low resistance vascular structure, ${ }^{9}$ a reduction of this force should increase OPA - provided the drug tested has no direct effect on ocular or systemic vascular systems. Surprisingly, vehicle treated contralateral control eyes showed a significant but less pronounced reduction in IOP, also noted in other primates ${ }^{23}$ which may be due to a systemic effect of dorzolamide; these eyes showed no change in OPA pointing to factors other than IOP to influence ocular haemodynamics in this range of IOPs.

Systemic perfusion variables being unaltered, Kiel et al ${ }^{24}{ }^{25}$ noted a negative correlation for IOP/choroidal blood flow $<25 \mathrm{~mm} \mathrm{Hg}$ in rabbits, postulating a myogenic regulation mechanism in the choroid reaching its adaptive maximum at $25 \mathrm{~mm} \mathrm{Hg}$.

Also, most other current glaucoma drugs reduce IOP but do not increase OPA ${ }^{1217}$ which may demonstrate a relative negative effect of these drugs on the choroidal vasculature and/or a compensatory mechanism for IOP changes in this vascular system in humans also.

A review of the literature shows contradictory results regarding the role of timolol with respect to ocular circulation. Timolol prevents a compensatory sympathetic reflex by blocking vascular $\beta_{2}$ and $\alpha$ receptors and thus may increase peripheral resistance which in turn may affect OPA, but this effect was not noted in our topical glaucoma drug studies. ${ }^{12}{ }^{17}$ Trew and Smith $^{26}$ noted no improvement in pulsatile ocular blood flow (POBF), a variable not calculated in our studies (see earlier), following application of timolol $0.25 \%$ in HTP patients whereas following application of timolol $0.5 \%$ Boles-Carenini et $a l^{27}$ showed a significant decrease of POBF; Martin and Rabineau ${ }^{28}$ noted a reduction of the mean retinal arterial diameter. In our studies the topical glaucoma drugs at the concentrations used in this study reduced IOP but did not affect OPA in ocular hypertensive volunteers ${ }^{12}$ and native cynomolgus monkey eyes. ${ }^{17}$

Dorzolamide additively increases the IOP lowering effect of timolol $^{29}{ }^{30}$; although the pharmacological interactions for adrenaline and dorzolamide are not yet described, owing to their different pharmacological modes of action (adrenaline, a direct $\alpha$ and $\beta$ sympathomimetic, reduces IOP by its $\alpha$ adrenergic vasoconstrictive effect on ciliary body capillaries, ${ }^{31}$ and $\alpha$ and $\beta$ adrenergic, probably cAMP mediated, ${ }^{32}$ increase in trabecular ${ }^{33}$ and uveoscleral outflow ${ }^{34}{ }^{35}$ ), it seems reasonable to also assume an additive IOP lowering effect.

Interactions of dorzolamide with timolol and/or adrenaline affecting OPA in the HTP group are conceivable but unlikely, owing to their different pharmacological modes of action and lack of effect of timolol and adrenaline on OPA when applied as monotherapy. ${ }^{12}$ In the limited study reported here there was no significant difference in IOP reduction, OPA increase, and systemic perfusion variables in dorzolamide/timolol $v$ dorzolamide/adrenaline HTP groups.

Measurements of IOP and OPA made during the course of development of the cynomolgus monkey glaucoma model gave the unexpected result that OPA does not decrease when IOP increases in the lasered eye, but instead it increased in the contralateral unlasered eye which responds with a higher OPA. This finding remains consistent after full 
development of the glaucoma. This result may seem paradoxical but is understandable if a higher IOP in one eye causes a systemic or bilateral compensatory regulation which is more manifest in the eye with normal IOP than in the lasered eye with the increased IOP. $^{17}$

Compensatory effects would seem to be limited to the choroid because lasered monkey eyes show no changes in the autoregulated retinal $^{36}$ or optic nerve head blood flow. ${ }^{37}$

This postulate is also in line with indications from studies in human subjects that glaucomatous eyes may have an impaired compensatory OPA response to IOP elevation, ${ }^{1-417}$ which may exhibit an impeded functional reserve in the choroid and may reflect a primary decrease in choroidal capacity, ${ }^{124}$ decreased perfusion pressures as measured by oculo-oscillodynamography, ${ }^{38}$ and/or reduced ophthalmic artery perfusion. ${ }^{39} 40$

Physical exercise in normal healthy volunteers significantly $(\mathrm{p}<0.05)$ increased heart rate $(208.0 \%)$, systolic blood pressure $(20.4 \%)$, and ocular perfusion pressure $(265.0 \%)$, and significantly $(\mathrm{p}<0.05)$ reduced IOP $(32.3 \%)$ and diastolic blood pressure $(17.0 \%)$. OPA was unchanged ${ }^{3}$ again suggesting functional isolation-that is, significant autoregulatory capacity of the choroidal vasculature in the normal range of IOPs of healthy volunteers, which appears to compensate for a significant increase in pulse pressure and the significant reduction in IOP.

Although we cannot rule out with absolute certainty an IOP/OPA interdependence for the IOP range examined, these results make it very unlikely that a change in IOP may account for the increase in OPA following application of dorzolamide.

Kitazawa et $a l^{5}$ demonstrated visual field improvements in six of 25 NTP patients following application of nifedipine, a calcium channel blocker, vasodilator, and systemic antihypertensive agent which is successfully used in vasospastic disorders. Netland et al ${ }^{41}$ observed a significant decrease in the progression of NTP but not in HTP over a 3.4 year period.

NTP patients with a vasospastic reaction treated with nifedipine, showed a significant $(\mathrm{p}<0.001)$ increase in OPA while NTP patients without a vasospastic reaction failed to show significant changes in OPA. ${ }^{4}$ The response to nifedipine in half of the NTP patients match the findings of Kitazawa et $a l^{5}$ who demonstrated that half of their POAG patients independent of IOP suffer from vasospastic disorders.

An increase in OPA without affecting $\mathrm{IOP}^{3}$ might explain the stabilising effect of nifedipine on visual fields in NTP observed by Netland. ${ }^{41}$ This effect is probably due to the calcium channel blocker's pharmacological mode of action. Calcium channel blockers primarily reduce arterial resistance by blocking calcium influx into the vascular smooth muscle cell ${ }^{42}$ and by inhibition of the endothelial vasoconstrictor endothelin- $1^{43}{ }^{44}$ elevated in peripheral blood samples of NTP patients. ${ }^{45} \mathrm{Up}$ to now calcium channel blockers were the only drugs known to increase OPA in a subgroup of glaucoma patients. ${ }^{3}$

CAIs are located in brain arteries ${ }^{46}$ and the capillaries of the choroid and retina ${ }^{47}$ Inhibition of this enzyme, predominantly by a change in $\mathrm{pH}$ resulting in metabolic acidosis, ${ }^{16}$ reduces resistance in large cerebral arteries and thus dilates these vessels. ${ }^{46}$

The increase in OPA following application of a topical CAI may be a reflection of this effect on the choroidal vasculature and is in keeping with the positive haemodynamic effects of this drug on retrobulbar, retinal, and epipapillary circulation observed by Harris et $a l,{ }^{48}$ is in line with similar findings reported for a systemic $\mathrm{CAI},{ }^{15}$ and confirms the theory for the CAI mode of action proposed by Pfeiffer et al. ${ }^{19}$ They consider that an improvement in visual function following application of systemic CAIs is due to increased ocular perfusion as a result of acidification of blood and tissues.

Although the clinical aspects of POAGs, a syndrome of progressive optic neuropathy, characterised by an unphysiologically large cup/disc ratio with reduced retinal sensitivity, visual field defects, and additional psychophysical alterations, ${ }^{49}$ are known in detail, the exact pathogenetic mechanism(s) resulting in this specific form of optic nerve head damage remain(s) unknown, it has become evident that glaucoma is a multifaceted disease. Attention to IOP reduction remains important, but does not address all mechanisms present within many POAG patients. Future medication must also increase perfusion to the eye.

As with all medications for glaucoma therapy, the ability of topical CAIs and other (selectively ocular) vasodilators to preserve visual function must be assessed in large studies performed over many years. Nevertheless, the limited study reported here indicates that vasoactive drugs may have potential in the specific treatment of POAG patients with reduced OPA.

Financial support: None.

Proprietary interest: None.

1 Schmidt KG, Stegmann DY, Serle JB, et al. Ocular pulse amplitude (OPA) in primary open angle glaucoma, low tension glaucoma, and in ocular hypertensive patients before and after topical drug treatment. Invest Ophthalmol Vis Sci 1991;32(Suppl): 943 .

2 Schmidt KG, v Rückmann A, Mittag TW. Okuläre Pulsamplitude bei okulärer Hypertension und verschiedenen Glaukomformen. Ophthalmologica 1998;212:5-10.

3 Schmidt KG, Mittag TW, Pavlovic S, et al. Influence of Schmidt KG, Mittag TW, Pavlovic S, et al. Influence of
physical exercise and nifedipine on ocular pulse amplitude. physical exercise and nifedipine on ocular pulse ampliti

Graefes Arch Clin Exp Ophthalmol 1996;234:527-32.
4 Schmidt KG, v Rückmann A, Mittag TW, et al. Reduced ocular pulse amplitude in low tension glaucoma is independent of vasospasm. Eye 1997;11:485-8.

5 Kitazawa Y, Shirai H, Go FJ. The effect of a Ca++ antagonist on visual field in low tension glaucoma. Graefes Arch Clin Exp Ophthalmol 1989;227:408-12.

6 Langham ME, To'Mey KF. A clinical procedure for the measurements of the ocular pulse-pressure relationship and ophthalmic arterial pressure. Exp Eye Res 1978;27:1725 .

7 Silver DM, Farrell RA. Validity of pulsatile ocular blood flow measurements. Surv Ophthalmol 1994;38(Suppl):7280 .

8 Alm A. Physiologie der okulären Durchblutung. In: Stodtmeister R, Christ T, Pillunat LE, Ulrich WD, eds. Okuläre Durchblutungsstörungen. 1st ed. Stuttgart: Enke,

9 Williamson TH, Harris A. Ocular blood flow measurement. Br f Ophthalmol 1994;78:939-45.

10 Butt Z. Reproducibility of pulsatile ocular blood flow measurement. f Glaucoma 1995;4:214-8. 
11 Chidlow G. The ocular blood flow tonometer: a new instrument for the measurement of intraocular pressure. Exp Eye Res 1996;63:463-9.

12 Gambhir S, Inao S, Todokoro M, et al. Comparison of vasodilatory effect of carbon dioxide inhalation and intravenous acetazolamide on brain vasculature using positron emission tomography. Neurol Res 1997;19:139-44.

13 Nighoghossian N, Berthezene Y, Meyer R, et al. Assessment of cerebrovascular reactivity by dynamic susceptibility contrast-enhanced MR imaging. F Neurol Sci 1997;149. 171-6.

14 Hayashida K, Tanaka Y, Hirose Y, et al. Vasoreactive effect of azetazolamide as a function of time with sequential PET 150-water measurement. Nucl Med Commun 1996;17: $1047-51$

15 Langham ME, McHenry M. The prevention of visual loss by ocular hypotensive drugs. Invest Ophthalmol Vis Sci 1995;36(Suppl):966.

16 Pfeiffer N. The potential for topical carbonic anhydrase inhibitors in glaucoma therapy. Curr Opin Ophthalmol 1994:5:20-5.

17 Mittag TW, Serle J, Schumer R, et al. Studies of the ocular pulse in primates. Surv Ophthalmol 1994;38(Suppl):18390 .

18 Lippa EA, von Deffner HA, Hoffmann HM, et al. Local tolerance and activity of MK-927, a novel topical carbonic anhydrase inhibitor. Arch Ophthalmol 1988;106:1694-6.

19 Pfeiffer N, Hennekes R, Lippa EA, et al. A single dose of the topical carbonic anhydrase inhibitor MK-927 decreases IOP in patients. Br $\mathcal{F}$ Ophthalmol 1990;74:405-8.

20 Higginbotham EJ, Kass MA, Lippa EA, et al. MK-927: a topical carbonic anhydrase inhibitor. Dose response and duration of action. Arch Ophthalmol 1990;108:65-8.

21 Serle JB, Lustgarten JS, Lippa EA, et al. MK-927, a topical carbonic anhydrase inhibitor. Dose response and reproducibility. Arch Ophthalmol 1990;108:838-41.

22 Lippa EA, Aasved H, Airaksinen PJ, et al. Multiple dose, dose response relationship for the topical carbonic dose response relationship for the topical carbonic
anhydrase inhibitor MK-927. Arch Ophthalmol 1991;109: 46-9.

23 Wang RF, Serle JB, Podos SM, et al. MK-507 (L-671, 152), a topically active carbonic anhydrase inhibitor, reduces aqueous humor production in monkeys. Arch Ophthalmo 1991;109:1297-9.

24 Kiel JW, Shepard AP. Autoregulation of choroidal blood flow in the rabbit. Invest Ophthalmol Vis Sci 1992;33:2399410

25 Kiel JW. Choroidal myogenic autoregulation and intraocular pressure. Exp Eye Res 1994;58:529-43.

26 Trew DR, Smith SE. Postural studies in pulsatile ocular blood flow: II. Chronic open angle glaucoma. $\mathrm{Br} f$ Ophthalmol 1991;75:71-5.

27 Boles-Carenini A, Sibour G, Boles-Carenini B. Differences in the long-term effect of timolol and betaxolol on the pulsatile ocular blood flow. Surv Ophthalmol 1994;8(Suppl): $118-24$.

28 Martin XD, Rabineau PA. Vasoconstrictive effect of topical timolol on human retinal arteries. Graefes Arch Clin Exp Ophthalmol 1989;227:526-30.

29 Hartenbaum D. The efficacy of dorzolamide, a topical carbonic anhydrase inhibitor, in combination with timolol in the treatment of patients with open-angle glaucoma and the treatment of patients with open-angle glauc

30 Heijl A, Strahlman E, Sverrisson T, et al. A comparison dorzolamide and timolol in patients with pseudoexfoliation and glaucoma or ocular hypertension. Ophthalmology 1997; 104:137-42.

31 Van Burskirk EM. The ciliary vasculature and its pertubation with drugs and surgery. Trans Am Ophthalmol Soc 988;86:794-841

32 Neufeld AH, Sears ML. Adenosine 3'5'-monophosphate ncreases the outflow of aqueous humor from the rabbit eye. Invest Ophthalmol Vis Sci 1981;20:371-81.

33 Sears ML. Autonomic nervous system:adrenergic agonists. In: Sears ML, ed. Handbook of experimental pharmacology. vol 69. Berlin: Springer, 1984.

34 Townsend DJ, Brubacker RF. Immediate effect of epinephrine on aqueous formation in the normal human eve as mhrine on aqueous formation in the normal human eye as 1980;19:256-66.

35 Nagataki S, Brubacker RF. Early effect of epinephrine on aqueous formation in the normal human eye. Invest Ophthalmol 1981;88:278-82.

36 Grunwald JE, Riva CE, Stone RA, et al. Retinal autoregulation in open-angle glaucoma. Ophthalmology 1984;91: 1690-4.

37 Quigley HA, Hohmann RM, Sanchez R, et al. Optic nerve head blood flow in chronic experimental glaucoma. Arch Ophthalmol 1985;103:956-62.

38 Pillunat LE, Stodtmeister R, Marquardt R, et al. Ocular perfusion pressures in different types of glaucoma. Int Ophthalmol 1989;13:37-42.

39 Harris A, Sergott, RC, Spaeth GL, et al. Color doppler analysis of ocular vessel blood velocity in normal-tension analysis of ocular vessel blood velocity in no.

40 Rojanapongpun P, Drance SM, Morrison BJ. Ophthalmic artery flow velocity in glaucomatous and normal subjects. Br f Ophthalmol 1993;77:25-9.

41 Netland PA, Chaturvedi N, Dreyer EB. Calcium channel blockers in the management of low tension and open-angle glaucoma. Am f Ophthalmol 1993;115:608-13.

42 Shrier I, Magder S. Effects of nifedipine on vascular waterfall and arterial resistance in canine hindlimb. Am f Physiol 1995;268:371-6.

43 Nyborg NCB, Prieto D, Benedito S, et al. Endothelin-1 induced contraction of bovine retinal small arteries is reversible and abolished by nitrendipine. Invest Ophthalmol Vis $S_{c i}$ 1991:32:27-31.

44 Harada K, Miwa A, Yokoyama T, et al. Pharmacological analysis of the inhibitory effects of KRN2391 on endothelin-1-induced contraction in isolated large coronary artery of the pig. Gen Pharmacol 1994;24:935-9.

45 Moriya S, Sugiyama T, Shimizu K, et al. Low-tension glaucoma and endothelin (ET-1). Folia Ophthalmol fpn 1992;43:554-9.

46 Postiglione A, Bobkiewicz T, Vinholdt-Pedersen E, et al. Cerebrovascular effects of angiotensin converting enzyme nhibition involve large artery dilation in rats. Stroke 1991; 22:1363-8.

47 Ridderstrale Y, Wistrand PJ, Brechue WF. Membraneassociated CA activity in the eye of the CA II-deficient mouse. Invest Ophthalmol Vis Sci 1994;35:2577-84.

48 Harris A, Arend O, Arend S, et al. Effects of topical dorzolamide on retinal and retrobulbar hemodynamics. Acta Ophthalmol 1996;74:569-72.

49 Flammer J. To what extent are vascular factors involved in the pathogenensis of glaucoma. In: Kaiser HJ, Flammer J, Hendrickson P, eds. Ocular blood flow. Glaucoma meeting, 1995. Basel: Karger, 1996:12-39. 